\title{
Editorial
}

\section{Advances in Friction Welding}

\author{
Wenya Li, ${ }^{1}$ Achilleas Vairis, ${ }^{2}$ and R. Mark Ward ${ }^{3}$ \\ ${ }^{1}$ Shaanxi Key Laboratory of Friction Welding Technologies, School of Materials Science and Engineering, \\ Northwestern Polytechnical University, Xian, Shaanxi 710072, China \\ ${ }^{2}$ Department of Mechanical Engineering, School of Technology, TEI of Crete, P.O. Box 1939, Estavromenos, 71004 Heraklion, Greece \\ ${ }^{3}$ School of Metallurgy and Materials, University of Birmingham, Birmingham B15 2TT, UK
}

Correspondence should be addressed to Wenya Li; liwy@nwpu.edu.cn

Received 3 June 2014; Accepted 3 June 2014; Published 3 July 2014

Copyright (C) 2014 Wenya Li et al. This is an open access article distributed under the Creative Commons Attribution License, which permits unrestricted use, distribution, and reproduction in any medium, provided the original work is properly cited.

There is a continual requirement to make things perform better, be more predictably reliable, cost less, and use less energy. This often leads to requirements to make components for them that are complex shapes or that have properties that vary from one location to another. These requirements are often difficult or expensive to satisfy by conventional machining and mechanical processing, but joining allows us to make complex shapes, potentially of materials with different properties. The disadvantage is that, often, joints require mass to be added to a design to carry the locally increased forces surrounding the joint. Alternatively the joint must be prepared and produced very carefully and may still have properties below those of the parent material. Extra mass or reduced properties (again requiring extra mass) increase the raw material costs and typically reduce the energy efficiency of the final product.

Friction welding offers an extremely promising way to make joints with minimal requirements for extra mass and with extremely good final product properties showing very low distortion. Particularly for metals appealing advantages are the self-cleaning nature of the process as it expels surface contamination that might ultimately lead to, for example, fatigue damage in service and the avoidance of melting at the joining interface which reduces metallurgical problems. All processes, to varying degree of course, are very reliable and repeatable, with few requirements in terms of nondestructive testing usually.

Considerable work on friction welding has been undertaken at the Universities of Birmingham, Manchester, and Cranfield, TWI (UK), Northwestern Polytechnical University
(China), Wroclaw University of Technology (Poland), University of Johannesburg (South Africa), University of Ulsan (South Korea), and Universiti Malaysia Perlis (Malaysia), among others. Inertia and rotary friction welding have been used since World War II in a wide range of applications such as shafts and valves, while linear friction welds are already being used in high performance parts in aircraft engines. The friction stir welding family of applications (friction stir welding, friction stir spot welding, friction stir processing, etc.) is used in the manufacture of structures utilising aluminium alloy plates of every grade.

The seven papers in this special edition cover a wide range of applications of the friction welding family ranging from rotary/inertia and linear to stir and stir spot welding, as well as friction stir processing. The topics of these papers are about dissimilar material joints, joint microstructure, mechanical properties, and various modelling techniques. We hope that you will find them useful in deciding how friction joining can be used in your applications.

Wenya Li

Achilleas Vairis

R. Mark Ward 

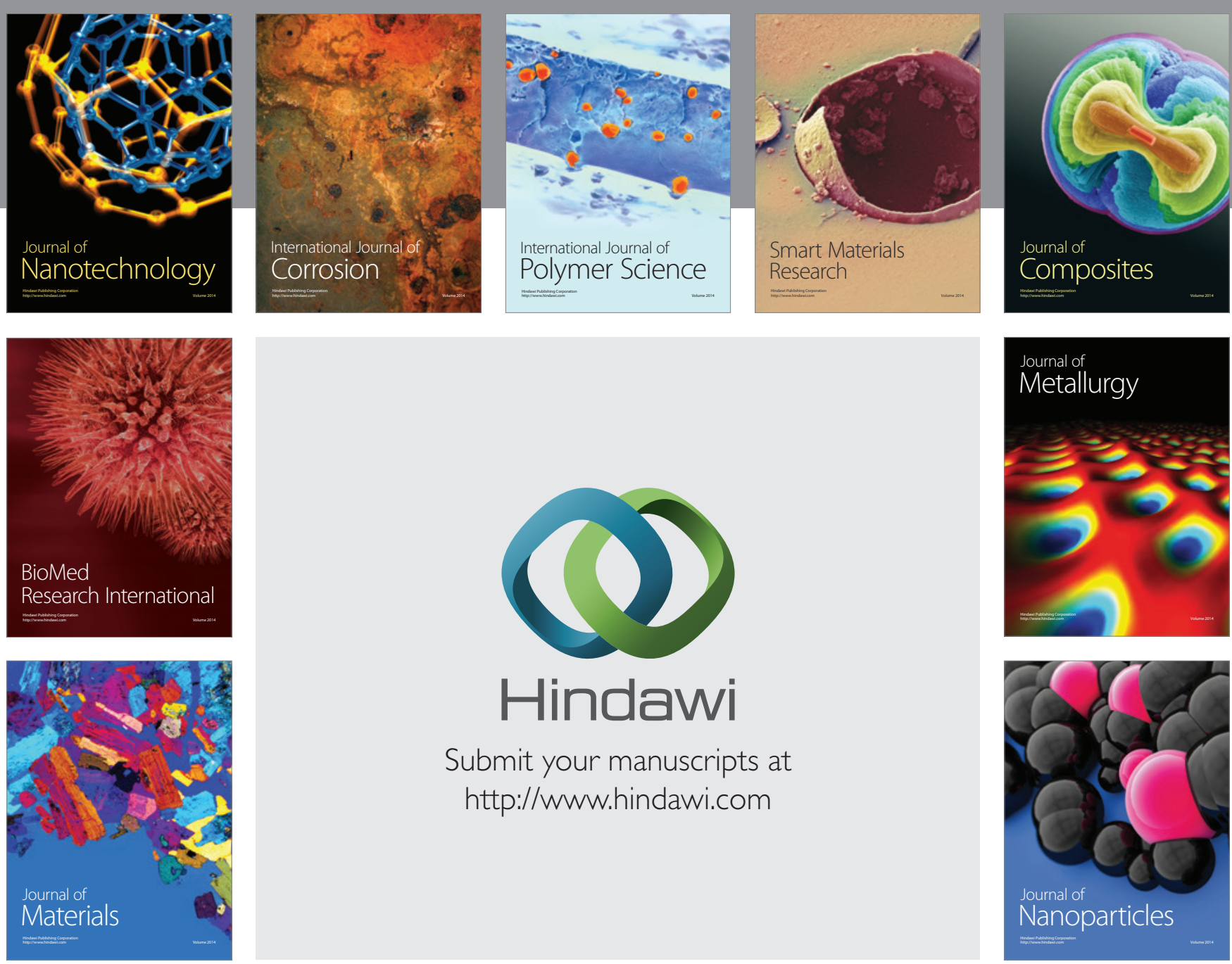

Submit your manuscripts at http://www.hindawi.com
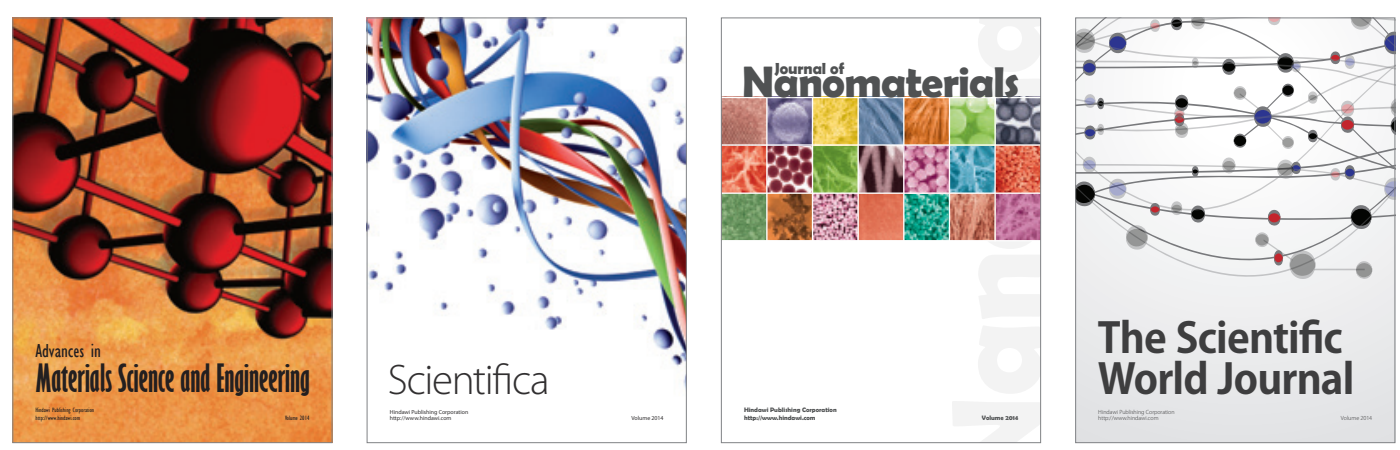

\section{The Scientific World Journal}
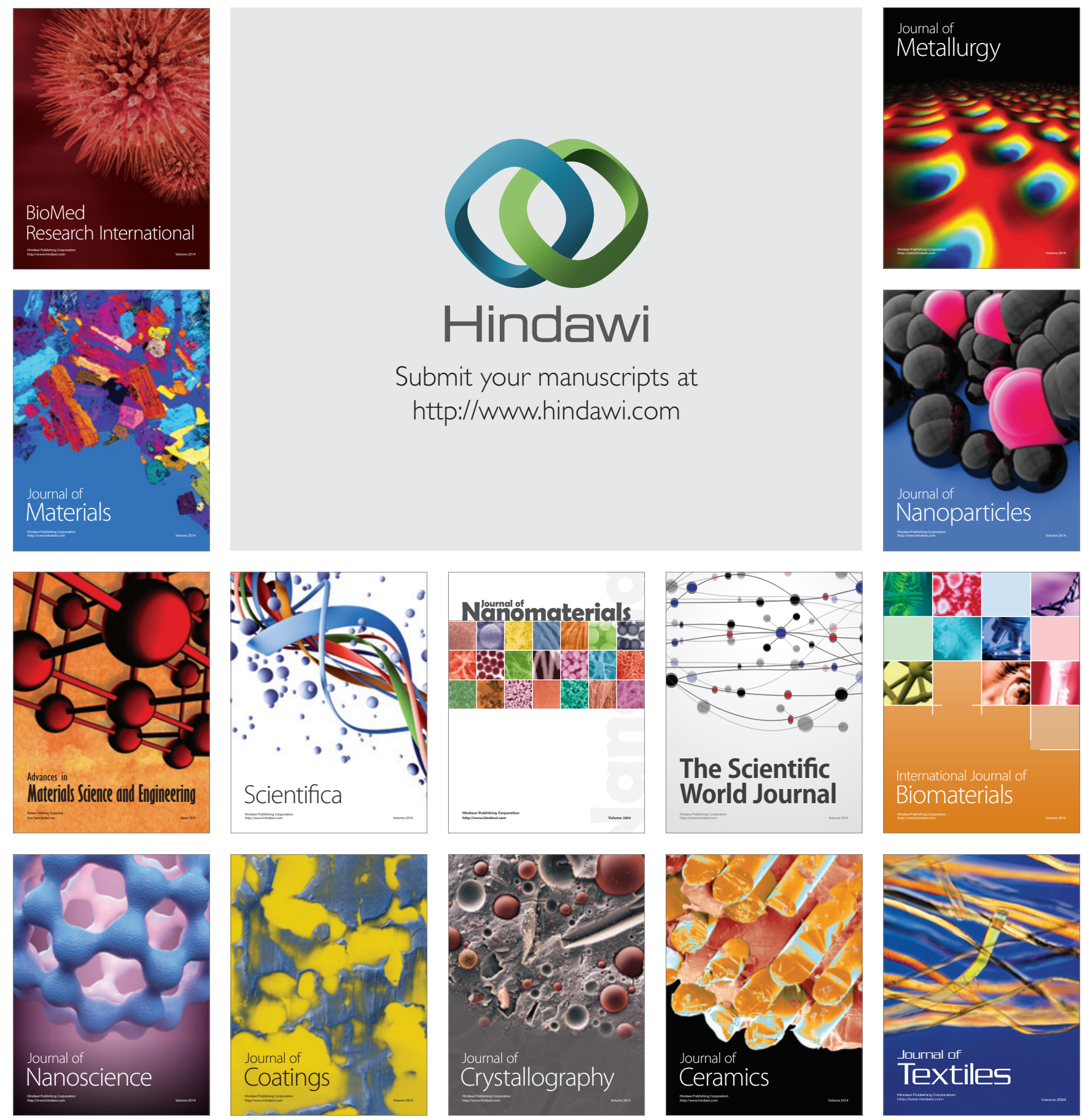